\title{
Estimating the Local Gas Pressure in a Gas Flow Cell Stage In Situ Using Electron Energy Loss Spectroscopy
}

\author{
R. Colby ${ }^{1}$, B. Kabius ${ }^{1}$, D.H. Alsem ${ }^{2}$ \\ 1. Environmental and Molecular Sciences Laboratory, Pacific Northwest National Laboratory, Richland, WA \\ 2. Hummingbird Scientific, Lacey, WA USA
}

The development of environmental transmission electron microscopy (TEM) has enabled in situ experiments in a gaseous environment with high resolution imaging and spectroscopy for many systems. Many important systems, in such areas as catalysis and geochemistry, require much higher pressures than the 20 mbar achievable with a differentially pumped, dedicated environmental TEM. Gas flow stages, in which the gaseous environment is contained between two thin membrane windows (typically $\mathrm{SiN}$ ), have been demonstrated at reported pressures of up to several atmospheres (Fig. 1a,b). While the potential to work at realistic pressures is attractive, the design of many current gas flow stages is such that the pressure at the sample cannot necessarily be directly inferred from the pressure differential across the entire system. The flow rate at the sample can depend sensitively upon parameters such as the spacing between the SiN windows, which will vary (often significantly) each time the cell is sealed. Furthermore, the window spacing and pressure are interdependent in a typical two-window gas cell design. The flow rate of a gas through the cell-the critical measure for reactions in an in situ experiment - should be sensitively dependent upon both the channel size and the pressure.

The presence of a gas between the windows was confirmed by comparison of electron energy loss spectra (EELS) of both SiN windows in vacuum and a sealed stage with a $0.5 \mathrm{ccm}$ flow of nitrogen, evidenced by the appearance of minor shoulders at $\sim 8$ and $\sim 13 \mathrm{eV}$. These features are consistent with in situ EELS measurements of pure $\mathrm{O}_{2}$ and $\mathrm{N}_{2}$ gasses [1]. Flow through the holder was confirmed by the simple expedient of submerging the exit line in water with a surface open to atmosphere, and observing a stream of bubbles. The gas cell is sealed in open air, which could explain the presence of $\mathrm{O}_{2}$ : but as the shoulder at $\sim 8 \mathrm{eV}$ had not diminished after several hours of flowing ultrapure nitrogen gas, this raises concerns as to the actual flow rate of the gas at the sample.

We have used EELS to more accurately diagnose the pressure in a gas flow stage at the sample, by assessing the electron inelastic mean free path through the gas flow stage for a range of input gas flow rates, in situ, within the TEM. The inelastic mean free path of an electron $(\lambda)$ within a given gas is directly related to the density of that gas. The spacing between the two gas windows

$(t)$ can be accurately determined by measuring the tone rings in the diffractogram of a defocused image through the two amorphous windows. The relationship between the pressure and the inelastic mean free path of the electron beam in a gas can be determined either by comparison to past measurements performed in a dedicated environmental TEM [2] or estimated by means of the ideal gas law.

With energy filtered imaging, these two tasks can be carried out simultaneously, quickly, and in a specific area of interest; either prior to or during an experiment; and with a relatively small 
electron dose. To emphasize the effect of inelastic scattering in the gas, experiments were performed at lower voltages $(80 \mathrm{kV})$. For nitrogen gas (Fig. 1c), there is an approximately linear increase $t / \lambda$ over the range of accessible flow rates. Measurement of roughly the same region of the $\mathrm{SiN}$ window in vacuum yields a $t / \lambda \approx 0.67$. These measurements suggest that under normal operating conditions, pressures at the sample may be significantly lower than $1 \mathrm{~atm}$. This approach is applicable for the other gasses that have typically been used for in situ TEM studies, as it depends only upon measuring the total inelastic:elastic scattering ratio and not a particular core-loss edge [3].

[1] Peter A. Crozier, Santhosh Chenna, In situ analysis of gas composition by electron energyloss spectroscopy for environmental transmission electron microscopy, Ultramicroscopy 111, 177-185 (2011)

[2] Joerg R. Jinschek, Stig Helveg, Image resolution and sensitivity in an environmental transmission electron microscope, Micron 43 (11), 1156-1168 (2012)

[3] A portion of the research was performed using EMSL, a national scientific user facility sponsored by the DOE-BER and located at PNNL, and with the support of the EMSL William Wiley postdoctoral fellowship. Portions were supported by the DOE, Office of Science, Division of Materials Sciences and Engineering under Award \#10122, and Division of Chemical Sciences under Award \#48526. A portion was carried out at NCEM, LBNL, under DOE-BES contract \#DE-AC02-05CH11231.

(a)

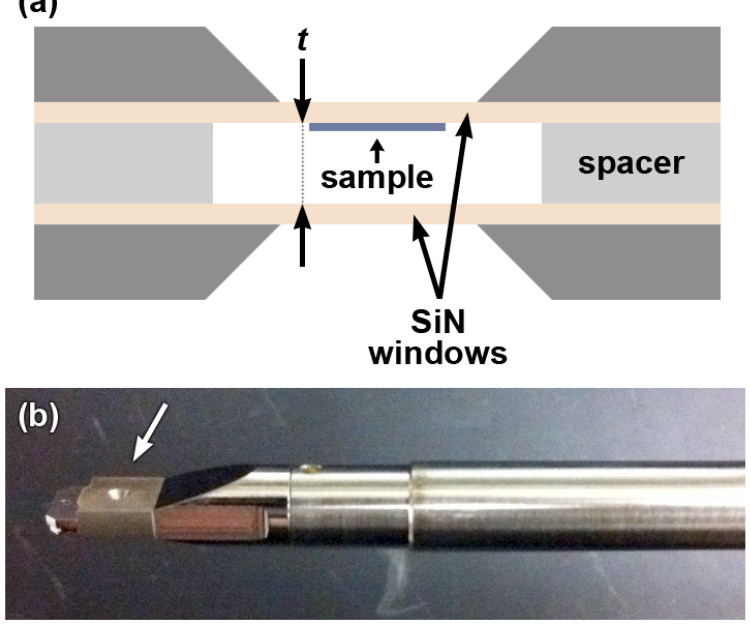

(c)

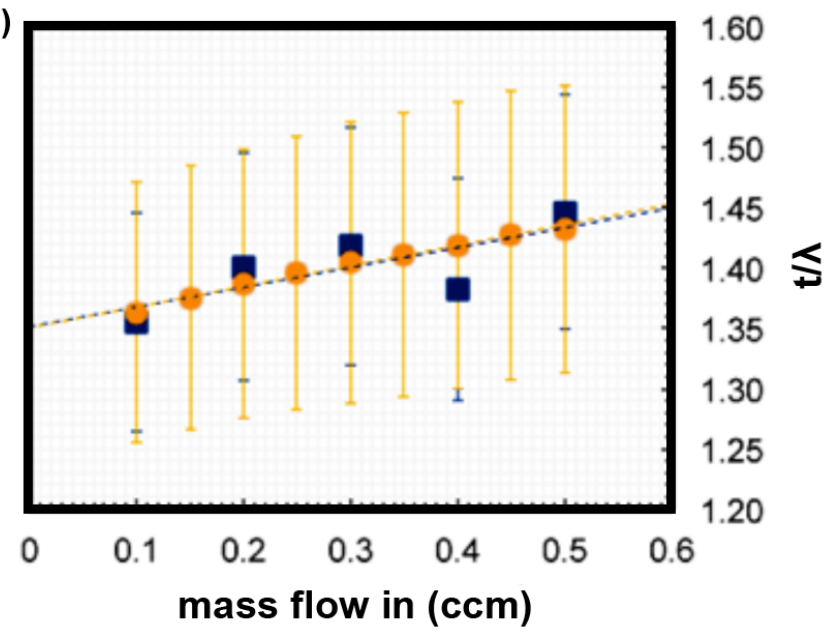

Figure 1: (a) schematic representation of the windows in a gas flow stage, separated a distance $(t)$ by a spacer, and (b) photograph of the tip of an actual gas flow stage. The white arrow points to the location of the windows. (c) the total ratio of the thickness over the inelastic mean free path $(t / \lambda)$, acquired by taking the natural $\log$ of the ratio of the total scattering and the elastically scattered electrons. The orange points represent the values while increasing the pressure, the blue squares while decreasing. Both are included to demonstrate that increase in $t / \lambda$ was not due, for instance, to an accumulation of beam induced reduction of contamination. Error bars were determined using the standard deviation of intensities across $2048 \times 2048$ pixel images with a $1 \mathrm{~s}$ total collection time, and can be significantly improved during collection, e.g. by binning. 